\title{
Mode decomposed chiral magnetic effect and rotating fermions
}

\author{
Kenji Fukushima, ${ }^{1, *}$ Takuya Shimazaki $\odot,{ }^{1, \dagger}$ and Lingxiao Wang $\odot^{2,1, *}$ \\ ${ }^{1}$ Department of Physics, The University of Tokyo, 7-3-1 Hongo, Bunkyo-ku, Tokyo 113-0033, Japan \\ ${ }_{2}^{2}$ Physics Department, Tsinghua University and Collaborative Innovation Center of Quantum Matter, \\ Beijing 100084, China
}

(Received 21 April 2020; accepted 19 July 2020; published 29 July 2020)

\begin{abstract}
We present a novel perspective to characterize the chiral magnetic and related effects in terms of angular decomposed modes. We find that the vector current and the chirality density are connected through a surprisingly simple relation for all the modes and any mass, which defines the mode decomposed chiral magnetic effect in such a way free from the chiral chemical potential. The mode decomposed formulation is useful also to investigate properties of rotating fermions. For demonstration, we give an intuitive account for a nonzero density emerging from a combination of rotation and magnetic field as well as an approach to the chiral vortical effect at finite density.
\end{abstract}

DOI: $10.1103 /$ PhysRevD.102.014045

\section{INTRODUCTION}

Chiral fermions exhibit fascinating transport properties, the origin of which is traced back to the quantum anomaly associated with chiral symmetry. Gauge theories including quantum chromodynamics (QCD) may accommodate topologically winding configurations and a chirality imbalance is induced [1,2]. Thanks to the index theorem, one can quantify the chirality change in response to the winding number even without solving QCD problems. There are some indirect evidences for the chiral anomaly such as the decay rate of $\pi^{0} \rightarrow 2 \gamma$, anomalously heavy $\eta^{\prime}$ meson [3,4], etc. It is still an ambitious problem to establish more direct experimental probes to the chiral anomaly [5].

The chiral magnetic effect (CME) is a well-investigated example of macroscopic realization of the chiral anomaly, which was originally proposed in the context of the relativistic heavy-ion collision experiment [6] (see also Ref. [7] for an earlier work) and an elegant formula with the chiral chemical potential $\mu_{5}$ was found in Ref. [8]. The CME generates a vector current $\boldsymbol{j}$ in parallel to an external magnetic field $\boldsymbol{B}$ in the presence of a finite chirality imbalance. In ordinary matter, such a vector current proportional to $\boldsymbol{B}$ is prohibited by parity and time-reversal symmetry, so the CME requires the chirality imbalance.

\footnotetext{
fuku@nt.phys.s.u-tokyo.ac.jp

shimazaki@nt.phys.s.u-tokyo.ac.jp

wlx15@tsinghua.org.cn
}

Published by the American Physical Society under the terms of the Creative Commons Attribution 4.0 International license. Further distribution of this work must maintain attribution to the author(s) and the published article's title, journal citation, and DOI. Funded by SCOAP.
In other words, the coefficient in front of $\boldsymbol{B}$ in the CME, i.e., the chiral magnetic conductivity [9], must be parity odd and time-reversal even. Interestingly, the CME is anomaly protected and the chiral magnetic conductivity at zero frequency is unaffected by higher-order corrections. There are a number of theoretical and experimental efforts to detect CME signatures; parity-odd fluctuations (called the $\gamma$-correlator) in the heavy-ion collisions [10-15] and the negative magnetoresistance in Weyl/Dirac semimetals [16-19] are representative examples.

A finite chirality imbalance plays a pivotal role in the CME, which supposedly results from topological excitations in the QCD case. For theoretical convenience, a finite chirality is imposed conventionally by $\mu_{5}$ coupled to the chirality charge. Sometimes, however, $\mu_{5}$ has caused controversies - the chemical potential is generally well defined for a conserved charge but the chirality charge is not conserved due to the chiral anomaly. Since it is not a conserved charge, $\mu_{5}$ must vanish in equilibrium. In other words, a finite $\mu_{5}$ as a theoretical device makes sense only out of equilibrium. It would be therefore desirable to setup the $\mathrm{CME}$ without $\mu_{5}$. In fact, a background electromagnetic field like $\boldsymbol{E} \propto \boldsymbol{B}$ is an alternative of $\mu_{5}$ used for experiments in Weyl/Dirac semimetals. For more details on the absence of the CME in equilibrium, see Ref. [20].

Let us turn to other intriguing phenomena in a chiral medium similar to the CME, namely, the chiral separation effect (CSE) [21-23] and the chiral vortical effect (CVE) [24-30]. In later discussions, we will shed light on the CSE and the CVE as well as the CME using the mode decomposed wave functions. In the CSE, an axial-vector current $\boldsymbol{j}_{5}$ is generated in parallel to an external magnetic field $\boldsymbol{B}$ in the presence of the chemical potential $\mu$. The CVE is quite analogous to the CSE; an axial-vector current 
is induced by a vorticity $\boldsymbol{\omega}$ instead of $\boldsymbol{B}$ [31-33]. Contrary to the CME, the CSE and the CVE are not anomaly protected and their coefficients could be affected by infrared scales such as the mass, the temperature, and so on [29,34-37]. Such a clear distinction between the CME and the CSE/CVE will be unraveled in our mode decomposed analysis. Our calculations will lead to a surprisingly simple relation between the vector current and the chirality density, which had been unseen until we made a mode decomposed formulation.

Interestingly, both strong magnetic field $\boldsymbol{B}$ and large vorticity $\boldsymbol{\omega}$ are highly relevant to the heavy-ion collisions (see Refs. [15,38] for recent reviews). Noncentral collisions would produce a quark-gluon plasma (or hot hadronic matter at lower collision energies) under strong $\boldsymbol{B}$ and large $\boldsymbol{\omega}[15,39-41]$. Now, chiral transport phenomena in general have grown up to be one major subject in not only heavyion collision but general physics $[42,43]$. So far, $\boldsymbol{B}$-induced and $\boldsymbol{\omega}$-induced phenomena have been considered individually, but an interplay between $\boldsymbol{B}$ and $\boldsymbol{\omega}$ would be becoming more and more attractive [44-52]. Among various theoretical attempts, we shall pay our special attention to Ref. [45] in which a finite density $\propto \boldsymbol{\omega} \cdot \boldsymbol{B}$ was discovered. The authors in Ref. [45] proposed redistribution of the vector charge induced by $\boldsymbol{\omega} \cdot \boldsymbol{B}$, which may carry a vital role in magnetohydrodynamics where $\boldsymbol{B}$ is too large to be regarded as the first order in the gradient expansion [27]. We will reproduce this result as a benchmark test of our formulation and discuss an intuitive picture to deepen our understanding on the induced density.

In this work, we shall intensively investigate fermion systems with $\boldsymbol{B}$ and analyze the mode decomposition. We will express the density, the chirality density, the vector current, and the axial-vector current in terms of the angular momentum modes. Our most prominent finding is an elegant mode-by-mode equation between the vector current $\boldsymbol{j}$ and the chirality density $\rho_{5}$, as we already mentioned above. Notably, the equation holds not only for the lowest landau levels (LLLs) but also higher Landau levels for any mass, while a similar equation between the axial-vector current $j_{5}$ and the density $\rho$ is valid for the LLLs only. The existence of such an elegant relation reminds us of the fact that the CME is anomaly protected. We should also emphasize that the anomaly nature of the CME appears from the LLL contribution, but the relation we found is for all Landau levels. We are proposing this mode decomposed $\mathrm{CME}$ as an extended interpretation of the CME without $\mu_{5}$. We note that we can straightforwardly recover the familiar formula of the CME by taking the mode sum with proper weights with $\mu_{5}$.

We can apply our mode decomposed formulation for rotating fermion systems. It is known that there is a reciprocal relationship between $\boldsymbol{B}$ and $\boldsymbol{\omega}$, but their microscopic descriptions are totally different. Once all angular modes are available, we can introduce $\boldsymbol{\omega}$ in the weight factor in a form of the chemical potential shift $[53,54]$. From this analogy between the rotational and finite-density effects, we can say that states with a certain angular momentum are reminiscent of those with a certain particle number in the "canonical" ensemble. The Legendre transformation gives the grand canonical ensemble with the chemical potential, and a similar machinery would work to translate the angular momentum modes into the "grand canonical" ensemble with a certain $\boldsymbol{\omega}$. One might think that we just recapitulate known calculations in the cylindrical coordinates, but our formulation can reveal properties of rotating fermions especially under $\boldsymbol{B}$ in an intuitive manner. As mentioned above, we will give a plain explanation of induced density discussed in Ref. [45], through which subtleties about the angular momentum associated with the magnetic field will be manifested.

Finally, let us briefly mention a possibility that our calculation may be directly applied to electron vortex beams in optics [55-62]. Unlike the topological vortex in a superfluid, what is called the fermion vortex is a fermion beam whose wave functions have a helical phase structure. For discussions from the view point of Berry phases, see Refs. [55,63-65]. It is also possible that light waves could have orbital angular momenta as speculated first by Poynting and this has been actually realized experimentally $[66,67]$.

The azimuthal dependence of electron vortex beams is $e^{i l \varphi}$ where $l \in \mathbb{Z}$ is a quantum number identified with the orbital angular momentum. Although a relativistic extension of the electron vortex beam is a theoretically challenging subject [68], nonrelativistic vortex beams have been observed experimentally and their applications have become an exciting research field [63,69-76]. In this work, we express various physical quantities using the angular momentum decomposed modes, and we can regard modeby-mode expressions as contributions from wave functions of vortex beams. Then, the mode decomposed CME as we advocate in this work should be directly tested with the vortex beams if the helicity of the vortex beams could be projected out. We can say, therefore, that we put forward an experimental possibility to realize chirally induced effects in tabletop setups in the future. It should be also emphasized that the fermion vortex beams have a lot of future prospects. The intrinsic orbital angular momentum of the vortex beams could take an arbitrarily large value in principle. For example, it has been reported that electron vortex beams up to $l \sim 1000$ (i.e., the orbital angular momentum $\sim 1000 \hbar$ ) have been experimentally observed [76]. Generally speaking, it is difficult to design experiments with large vorticity $\omega$ under reasonable control, but such states with large $l$ would provide us with an alternative and controllable probe to investigate rotating fermions.

Although our theoretical background lies in QCD physics, we will employ the convention of electrons and positrons in this work. If necessary, it would be easy to 
generalize our formulas for the purpose of QCD studies in terms of quarks by adjusting electric charges. Throughout this paper, we employ the physical units of $\hbar=c=1$.

\section{SOLUTIONS OF THE DIRAC EQUATION IN THE CYLINDRICAL COORDINATES}

We present explicit forms of the solutions of the Dirac equation in the cylindrical coordinates with the quantum number $l$ corresponding to the orbital angular momentum. Those expressions also explain our notations and conventions used throughout this paper.

The vacuum structures in quantum field theories should not be modified solely by rotation once the boundary effects are properly taken into account not to violate the causality $[25,44]$. In contrast, as demonstrated in Refs. [48,49,77,78], the vacuum structures would change if the fermionic wave functions are localized by coupling to external environments and thus become insensitive to the boundary effects. In this work, we shall utilize the cylindrical coordinates, $(r, \varphi, z)$, under a constant magnetic field, $\boldsymbol{B}=B \boldsymbol{e}_{z}$, so that the Landau wave functions are exponentially localized on the transverse $(r, \varphi)$ plane.

Throughout this work, we employ the Dirac representation for the $\gamma$ matrices, i.e.,

$$
\gamma^{0}=\left(\begin{array}{cc}
I & 0 \\
0 & -I
\end{array}\right), \quad \gamma^{i}=\left(\begin{array}{cc}
0 & \sigma_{i} \\
-\sigma_{i} & 0
\end{array}\right),
$$

where $I$ is the $2 \times 2$ unit matrix and $\sigma_{i}$ 's are the Pauli matrices. In this convention, $\gamma_{5}$ takes a nondiagonal form of

$$
\gamma_{5}=\left(\begin{array}{cc}
0 & I \\
I & 0
\end{array}\right)
$$

The solutions of the free Dirac equation with the electric charge convention, $e<0$, read in the Dirac representation,

$$
u_{n, l, k}^{(\uparrow)}(r, \varphi, z, t)=\frac{e^{-i \varepsilon_{n, l, k}^{(\uparrow)} t+i k z}}{\sqrt{\varepsilon_{n, l, k}^{(\uparrow)}+m}}\left(\begin{array}{c}
\left(\varepsilon_{n, l, k}^{(\uparrow)}+m\right) \Phi_{n, l}\left(\chi^{2}, \varphi\right) \\
0 \\
k \Phi_{n, l}\left(\chi^{2}, \varphi\right) \\
i \sqrt{|e| B(2 n+|l|+l+2)} \Phi_{n, l+1}\left(\chi^{2}, \varphi\right)
\end{array}\right)
$$

for the state with the positive helicity, the positive energy, and (the $z$ component of) the total angular momentum, $J_{z}=l+\frac{1}{2}$. In the above expression, $n$ and $l$ form the Landau level index and $l$ and $s$ represent the $z$-component eigenvalues of the orbital and the spin angular momenta, respectively. The $z$ component of the three momentum is denoted by $k$. A mixture of $l$ and $l+1$ in the spinor components stems from twofold realization of the same $J_{z}$ as $l+\frac{1}{2}$ and $(l+1)-\frac{1}{2}$. In the same way, the negative helicity and positive energy states read

$$
u_{n, l, k}^{(\downarrow)}(r, \varphi, z, t)=\frac{e^{-i \varepsilon_{n, l, k}^{(\downarrow)} t+i k z}}{\sqrt{\varepsilon_{n, l, k}^{(\downarrow)}+m}}\left(\begin{array}{c}
0 \\
-i \sqrt{|e| B(2 n+|l+1|+l+1)} \Phi_{n, l}^{(\downarrow)}\left(\chi^{2}, \varphi\right)
\end{array}\right)
$$

for the same total angular momentum, $J_{z}=l+\frac{1}{2}$. In the expressions (3) and (4), we introduced a dimensionless variable $\chi^{2}=\frac{1}{2} e B r^{2}$ and defined a function $\Phi_{n, l}\left(\chi^{2}, \varphi\right)$ as

$$
\Phi_{n, l}\left(\chi^{2}, \varphi\right)=\sqrt{\frac{n !}{(n+|l|) !}} e^{-\frac{1}{2} \chi^{2}} \chi^{|l|} L_{n}^{|l|}\left(\chi^{2}\right) e^{i l \varphi} .
$$

It would be useful to make it clear how we chose the normalization of the above function, i.e., our convention is

$$
\int d x d y\left|\Phi_{n, l}\left(\chi^{2}, \varphi\right)\right|^{2}=\frac{2 \pi}{|e B|} \int d \chi^{2}\left|\Phi_{n, l}\left(\chi^{2}, \varphi\right)\right|^{2}=\frac{2 \pi}{|e B|} .
$$

This Landau wave function, representing a localized spatial profile, has an exponential damping factor for large $r^{2}$. For these states of $u_{n, l, k}^{(\uparrow)}$ and $u_{n, l, k}^{(\downarrow)}$, the one particle eigenenergies are given by

$$
\left\{\begin{array}{l}
\varepsilon_{n, l, k}^{(\uparrow)}=\sqrt{|e| B(2 n+|l|+l+2)+k^{2}+m^{2}}, \\
\varepsilon_{n, l, k}^{(\downarrow)}=\sqrt{|e| B(2 n+|l+1|+l+1)+k^{2}+m^{2}} .
\end{array}\right.
$$


We note that the normalization (6) corresponds to the following convention for the Dirac spinor normalization:

$$
\frac{|e B|}{2 \pi} \int d x d y u_{n, l, k}^{(\uparrow, \downarrow) \dagger} u_{n, l, k}^{(\uparrow, \downarrow)}=2 \varepsilon_{n, l, k}^{(\uparrow, \downarrow)} .
$$

This is reduced to the standard normalization in the limit of $e B \rightarrow 0$, which can be understood from $\int d x d y e^{-\chi^{2}}=2 \pi /|e B|$. In later discussions, in fact, we will consider the $e B \rightarrow 0$ limit, which needs extra care about the treatment of $2 \pi /|e B|$ which is cut off by the transverse area $S_{\perp}$ for $e B \rightarrow 0$.

The antiparticle solutions of the free Dirac equation in the magnetic field with $J_{z}=l+\frac{1}{2}$ read

$$
v_{n, l, k}^{(\uparrow)}(r, \varphi, z, t)=\frac{e^{i \bar{\varepsilon}_{n, l, k}^{(\uparrow)} t-i k z}}{\sqrt{\bar{\varepsilon}_{n, l, k}^{(\uparrow)}+m}}\left(\begin{array}{c}
-i \sqrt{|e| B(2 n+|l|-l)} \Phi_{n,-l-1}\left(\chi^{2}, \varphi\right) \\
-k \Phi_{n,-l}\left(\chi^{2}, \varphi\right) \\
0 \\
\left(\bar{\varepsilon}_{n, l, k}^{(\uparrow)}+m\right) \Phi_{n,-l}\left(\chi^{2}, \varphi\right)
\end{array}\right)
$$

for the positive helicity antiparticle state. The antiparticle states with the negative helicity read

$$
v_{n, l, k}^{(\downarrow)}(r, \varphi, z, t)=\frac{e^{i \bar{\varepsilon}_{n, l, k}^{(\downarrow)} t i k z}}{\sqrt{\bar{\varepsilon}_{n, l, k}^{(\downarrow)}+m}}\left(\begin{array}{c}
-k \Phi_{n,-l-1}\left(\chi^{2}, \varphi\right) \\
-i \sqrt{|e| B(2 n+|l+1|-l+1)} \Phi_{n,-l}\left(\chi^{2}, \varphi\right) \\
-\left(\bar{\varepsilon}_{n, l, k}^{(\downarrow)}+m\right) \Phi_{n,-l-1}\left(\chi^{2}, \varphi\right) \\
0
\end{array}\right),
$$

which carries the same total angular momentum, $J_{z}=l+\frac{1}{2}$. The antiparticle eigenenergies are written as

$$
\left\{\begin{array}{l}
\bar{\varepsilon}_{n, l, k}^{(\uparrow)}=\sqrt{|e| B(2 n+|l|-l)+k^{2}+m^{2}}, \\
\bar{\varepsilon}_{n, l, k}^{(\downarrow)}=\sqrt{|e| B(2 n+|l+1|-l+1)+k^{2}+m^{2}} .
\end{array}\right.
$$

Now, for developing deeper understanding of magnetic properties, it is crucially important to see where the LLLs lie in this setup. For particles with $e<0$ (or the electrons particularly), we can immediately identify the LLLs as $(\downarrow)$ states with $n=0$ and $l \leq-1$ (i.e., $J_{z}<0$ ), for which $\varepsilon_{n=0, l \leq-1, k}^{(\downarrow)}=\sqrt{k^{2}+m^{2}}$. For antiparticles (or the

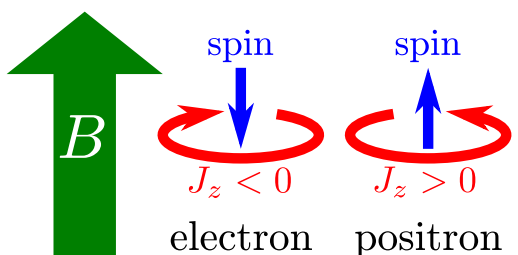

FIG. 1. Schematic illustration of the LLLs for negatively charged particles (electrons) and positively charged antiparticles (positrons). The preferred spin and orbital alignments are consistent with classical motion of charged particles. positrons), in the same way, the LLLs are found to be $(\uparrow)$ states with $n=0$ and $l \geq 0$ (i.e., $J_{z}>0$ ), for which $\bar{\varepsilon}_{n=0, l>0, k}^{(\uparrow)}=\sqrt{k^{2}+m^{2}}$. The range of $l$ is $(-\infty, \infty)$, but the LLLs span over $-S_{\perp}|e B| /(2 \pi)<J_{z}<0$ for the $(\downarrow)$ particles and $0<J_{z}<S_{\perp}|e B| /(2 \pi)$ for the $(\uparrow)$ antiparticles for sufficiently large $S_{\perp}|e B| /(2 \pi)$ (see Ref. [46] for details).

We note that the preferred $(\uparrow, \downarrow)$ and the range of $l$ for these LLLs are completely consistent with our intuition based on classical physics; the energy is lowered by the spin alignment antiparallel to $B$ for negatively charged particles, while the spin alignment should be parallel to $B$ for positively charged antiparticles. The orbital dynamics is in accord to the Larmor motion in classical electrodynamics and $J_{z}<0$ for electrons and $J_{z}>0$ for positrons are naturally concluded, respectively. For a schematic illustration, see Fig. 1.

\section{MODE DECOMPOSED DENSITY DISTRIBUTIONS}

We will consider mode-by-mode contributions to the expectation values of the scalar (density), the pseudoscalar (chirality density), the vector, and the axial-vector operators. We will later make use of the results in this section to propose the mode decomposed version of the CME. Also, we apply our results for a canonical formulation of rotating fermions. 


\section{A. Density and chirality density}

The fermion number density is given by $\rho=\left\langle\hat{\psi}^{\dagger} \hat{\psi}\right\rangle$, where $\hat{\psi}$ represents a Dirac field operator. It is a standard procedure to expand $\hat{\psi}$ in terms of the complete basis of $u_{n, l, k}^{(\uparrow, \downarrow)}$ with particle annihilation operators $\hat{a}_{n, l, k}^{(\uparrow, \downarrow)}$ and $v_{n, l, k}^{(\uparrow, \downarrow)}$ with antiparticle creation operators $\hat{b}_{n, l, k}^{(\uparrow, \downarrow) \dagger}$ in the second quantization method. All annihilation and creation operators are normal ordered. Subtleties of the normal-ordered operators were discussed in Refs. [79,80]. In this work, however, we consider one-particle states individually, so we have dropped infinite classical numbers from the anticommutation relation. Then, we can express $\rho$ as a linear superposition of different $(n, l, k)$ contributions, which we can symbolically represent in the following form:

$$
\rho=\sum_{n, l, k}\left(\rho_{n, l, k}^{(\uparrow)}+\rho_{n, l, k}^{(\downarrow)}+\bar{\rho}_{n, l, k}^{(\uparrow)}+\bar{\rho}_{n, l, k}^{(\downarrow)}\right),
$$

where the first two (and the last two) represent the particle (and the antiparticle) contributions. The sum integral is a shorthand representation of the phase space sum over $(n, l, k)$ with the proper weight having the mass dimension three. More explicitly, the phase space integral is replaced as

$$
\int \frac{d^{2} k_{\perp}}{(2 \pi)^{2}} \Leftrightarrow \frac{1}{S_{\perp}} \sum_{n, l}{ }^{\prime} \rightarrow \frac{|e B|}{2 \pi} \sum_{n, l}
$$

where $S_{\perp}$ is the transverse area and $\sum^{\prime}$ denotes a weighted sum that can reproduce the phase space integral in the zero magnetic limit and the weight goes to $S_{\perp}|e B| /(2 \pi)$ for sufficiently large $S_{\perp}|e B| /(2 \pi)$. For a precise definition, see Ref. [46]; we need to cope with a finite sized boundary condition and this is beyond the current scope.

These expectation values of $\rho_{n, l, k}^{(\uparrow, \downarrow)}$ and $\bar{\rho}_{n, l, k}^{(\uparrow, \downarrow)}$ depend on the state. If we take an expectation value with $\hat{a}_{n, l, k}^{(\uparrow) \dagger}|0\rangle$, we can immediately find the density constituent, using the explicit solutions of the Dirac equation, as

$$
\begin{aligned}
\rho_{n, l, k}^{(\uparrow)}= & \frac{\left(\varepsilon_{n, l, k}^{(\uparrow)}+m\right)^{2}+k^{2}}{2 \varepsilon_{n, l, k}^{(\uparrow)}\left(\varepsilon_{n, l, k}^{(\uparrow)}+m\right)}\left|\Phi_{n, l}\right|^{2} \\
& +\frac{|e| B(2 n+|l|+l+2)}{2 \varepsilon_{n, l, k}^{(\uparrow)}\left(\varepsilon_{n, l, k}^{(\uparrow)}+m\right)}\left|\Phi_{n, l+1}\right|^{2} .
\end{aligned}
$$

In the same way, we consider an expectation value corresponding to a state, $\hat{a}_{n, l, k}^{(\downarrow)}|0\rangle$, which turns out to be

$$
\begin{aligned}
\rho_{n, l, k}^{(\downarrow)}= & \frac{\left(\varepsilon_{n, l, k}^{(\downarrow)}+m\right)^{2}+k^{2}}{2 \varepsilon_{n, l, k}^{(\downarrow)}\left(\varepsilon_{n, l, k}^{(\downarrow)}+m\right)}\left|\Phi_{n, l+1}\right|^{2} \\
& +\frac{|e| B(2 n+|l+1|+l+1)}{2 \varepsilon_{n, l, k}^{(\downarrow)}\left(\varepsilon_{n, l, k}^{(\downarrow)}+m\right)}\left|\Phi_{n, l}\right|^{2} .
\end{aligned}
$$

Similarly, for the antiparticle contributions, states $\hat{b}_{n, l, k}^{(\uparrow, \downarrow) \dagger}|0\rangle$ lead to the expectation values as given by

$$
\begin{aligned}
\bar{\rho}_{n, l, k}^{(\uparrow)}= & -\frac{\left(\bar{\varepsilon}_{n, l, k}^{(\uparrow)}+m\right)^{2}+k^{2}}{2 \bar{\varepsilon}_{n, l, k}^{()}\left(\bar{\varepsilon}_{n, l, k}^{(\uparrow)}+m\right)}\left|\Phi_{n,-l}\right|^{2} \\
& -\frac{|e| B(2 n+|l|-l)}{2 \bar{\varepsilon}_{n, l, k}^{(\uparrow)}\left(\bar{\varepsilon}_{n, l, k}^{(\uparrow)}+m\right)}\left|\Phi_{n,-l-1}\right|^{2}
\end{aligned}
$$

and

$$
\begin{aligned}
\bar{\rho}_{n, l, k}^{(\downarrow)}= & -\frac{\left(\bar{\varepsilon}_{n, l, k}^{(\downarrow)}+m\right)^{2}+k^{2}}{2 \bar{\varepsilon}_{n, l, k}^{(\downarrow)}\left(\bar{\varepsilon}_{n, l, k}^{(\downarrow)}+m\right)}\left|\Phi_{n,-l-1}\right|^{2} \\
& -\frac{|e| B(2 n+|l+1|-l+1)}{2 \bar{\varepsilon}_{n, l, k}^{(\downarrow)}\left(\bar{\varepsilon}_{n, l, k}^{(\downarrow)}+m\right)}\left|\Phi_{n,-l}\right|^{2} .
\end{aligned}
$$

The overall minus sign of $\bar{\rho}$ comes from the anticommutation relation of $\hat{b}$. For our later quantitative discussions, it is important to note that the spatially integrated quantities (denoted by $\mathfrak{r}$ here) are, corresponding to Eq. (6), quantized as

$$
\begin{aligned}
& \mathfrak{r}_{n, l, k}^{(\uparrow, \downarrow)}=\int d x d y \rho_{n, l, k}^{(\uparrow, \downarrow)}=\frac{2 \pi}{|e B|}, \\
& \overline{\mathfrak{r}}_{n, l, l)}^{(\uparrow, \downarrow)}=\int d x d y \bar{\rho}_{n, l, k}^{(\uparrow, \downarrow)}=-\frac{2 \pi}{|e B|}
\end{aligned}
$$

for any $(n, l, k)$.

We make density plots for $\rho_{n, l, k}^{(\downarrow)}$ in Figs. 2 and 3 to visualize spatial distributions of the $s$-wave and $d$-wave
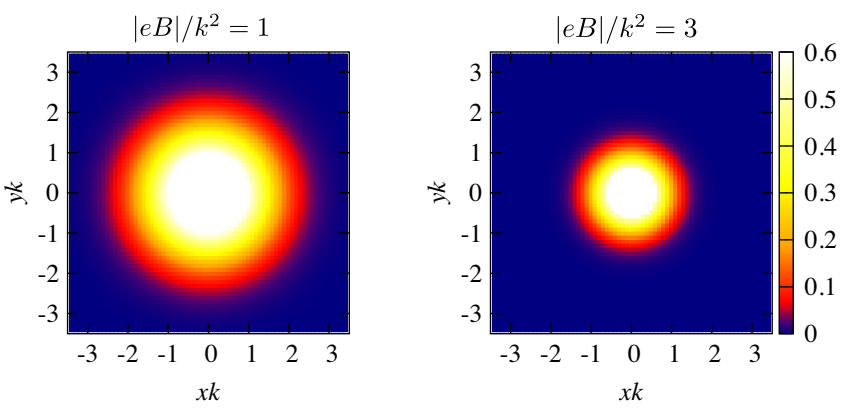

FIG. 2. Density plot of $\rho_{n, l, k}^{(\downarrow)}$ from one LLL with $n=0, l=-1$ (i.e., $J_{z}=-\frac{1}{2}$ ) with all dimensionful quantities rescaled with $k$. The mass is chosen as $m / k=1$. The left panel is for the magnetic strength $|e| B / k^{2}=1$ and the right one for $|e| B / k^{2}=3$. 

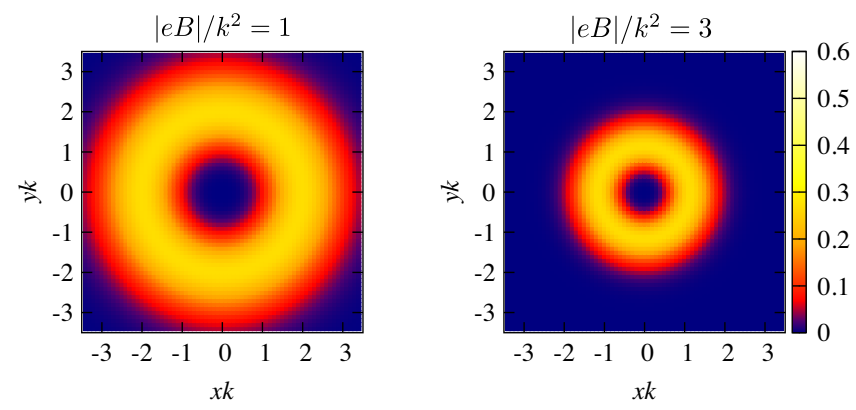

FIG. 3. Density plot of $\rho_{n, l, k}^{(\downarrow)}$ from another LLL with $n=0$, $l=-3$ (i.e., $J_{z}=-\frac{5}{2}$ ) with all dimensionful quantities rescaled with $k$. The mass is chosen as $m / k=1$. The left panel is for the magnetic strength $|e| B / k^{2}=1$ and the right one for $|e| B / k^{2}=3$.

LLL states (there are essentially the same plots shown in Ref. [59]). In our assignment $l=-1$ for the $\downarrow$ spin corresponds to the $s$-wave state with $J_{z}=-\frac{1}{2}$, so that the density is peaked around the origin in Fig. 2. As the magnetic field increases from the left panel to the right panel, the wave function becomes more localized near the origin, as is clear in both Figs. 2 and 3. Then, such a suppression factor by $1 /(e B)$ in Eq. (18) is naturally understood from reduction of distributed areas. Figure 3 is a plot for the $l=-3$ or $J_{z}=-\frac{5}{2}$ mode. In this case, the centrifugal force makes the wave function peaks farther from the origin and there is a hollow around the origin.

Next, we shall consider the chirality density, i.e., $\rho_{5}=\left\langle\hat{\psi}^{\dagger} \gamma_{5} \hat{\psi}\right\rangle$, in the same way, which can be symbolically decomposed again as

$$
\rho_{5}=y_{n, l, k}\left(\rho_{5 n, l, k}^{(\uparrow)}+\rho_{5 n, l, k}^{(\downarrow)}+\bar{\rho}_{5 n, l, k}^{(\uparrow)}+\bar{\rho}_{5 n, l, k}^{(\downarrow)}\right) .
$$

Here, simple calculations immediately lead to the following expressions:

$\rho_{5 n, l, k}^{(\uparrow)}=\frac{k}{\varepsilon_{n, l, k}^{(\uparrow)}}\left|\Phi_{n, l}\right|^{2}, \quad \rho_{5 n, l, k}^{(\downarrow)}=-\frac{k}{\varepsilon_{n, l, k}^{(\downarrow)}}\left|\Phi_{n, l+1}\right|^{2}$

for $\hat{a}_{n, l, k}^{(\uparrow, \downarrow) \dagger}|0\rangle$ states and

$\bar{\rho}_{5 n, l, k}^{(\uparrow)}=\frac{k}{\bar{\varepsilon}_{n, l, k}^{(\uparrow)}}\left|\Phi_{n,-l}\right|^{2}, \quad \bar{\rho}_{5 n, l, k}^{(\downarrow)}=-\frac{k}{\bar{\varepsilon}_{n, l, k}^{(\downarrow)}}\left|\Phi_{n,-l-1}\right|^{2}$

for $\hat{b}_{n, l, k}^{(\uparrow, \downarrow) \dagger}|0\rangle$ states. These are surprisingly simple expressions as compared to counterparts of the fermion number density. Unlike the fermion density, we see that the net chirality is vanishing after taking the mode sum over $k$. This is because the combination of $(\uparrow, \downarrow)$ and $k$ uniquely fixes whether the chirality is positive or negative. Usually for massless fermions, the chirality is determined by the spin and the momentum directions; in the present setup, $k$ is nothing but the momentum direction and $(\uparrow, \downarrow)$ corresponds to the spin direction. Supposing that states with a particular $k \neq 0$ are prepared, LLLs are states with the largest chirality, i.e.,

$$
\begin{aligned}
& \mathfrak{r}_{5, \mathrm{LLL}}^{(\downarrow)}=\int d x d y \rho_{5, \mathrm{LLL}}^{(\downarrow)}=-\frac{2 \pi}{|e B|} \frac{k}{\varepsilon_{k}}, \\
& \overline{\mathfrak{r}}_{5, \mathrm{LLL}}^{(\uparrow)}=\int d x d y \bar{\rho}_{5, \mathrm{LLL}}^{(\uparrow)}=\frac{2 \pi}{|e B|} \frac{k}{\varepsilon_{k}}
\end{aligned}
$$

after the spatial integration, where $\varepsilon_{k}=\sqrt{k^{2}+m^{2}}$. In the massless limit of $m \rightarrow 0$, we see that $k / \varepsilon_{k}$ reduces to the sign function of $k$.

In this paper, we would not plot $\rho_{5 n, l, k}^{(\uparrow, \downarrow)}$ nor $\bar{\rho}_{5 n, l, k}^{(\uparrow, \downarrow)}$, for they look indistinguishably similar to Figs. 2 and 3 on the qualitative level.

\section{B. Vector and axial vector currents}

We can further proceed to the vector and the axial vector currents, i.e., $j^{z}=\left\langle\hat{\psi}^{\dagger} \gamma^{0} \gamma^{z} \hat{\psi}\right\rangle$ and $j_{5}^{z}=\left\langle\hat{\psi}^{\dagger} \gamma^{0} \gamma^{z} \gamma_{5} \hat{\psi}\right\rangle$ mode by mode. Interestingly, for the vector components, we find such simple expressions as

$$
j_{n, l, k}^{z(\uparrow)}=\frac{k}{\varepsilon_{n, l, k}^{(\uparrow)}}\left|\Phi_{n, l}\right|^{2}, \quad j_{n, l, k}^{z(\downarrow)}=\frac{k}{\varepsilon_{n, l, k}^{(\downarrow)}}\left|\Phi_{n, l+1}\right|^{2}
$$

for $\hat{a}_{n, l, k}^{(\uparrow, \downarrow) \dagger}|0\rangle$ states and

$$
\bar{j}_{n, l, k}^{z(\uparrow)}=-\frac{k}{\bar{\varepsilon}_{n, l, k}^{(\uparrow)}}\left|\Phi_{n,-l}\right|^{2}, \quad \bar{j}_{n, l, k}^{z(\downarrow)}=-\frac{k}{\bar{\varepsilon}_{n, l, k}^{(\downarrow)}}\left|\Phi_{n,-l-1}\right|^{2}
$$

for $\hat{b}_{n, l, k}^{(\uparrow, \downarrow) \dagger}|0\rangle$ states. Here, it is a quite interesting and profound observation that the following relations should hold:

$$
\begin{array}{ll}
j_{n, l, k}^{z(\uparrow)}=\rho_{5 n, l, k}^{(\uparrow)}, & j_{n, l, k}^{z(\downarrow)}=-\rho_{5 n, l, k}^{(\downarrow)}, \\
\bar{j}_{n, l, k}^{z(\uparrow)}=-\bar{\rho}_{5 n, l, k}^{(\uparrow)}, & \bar{j}_{n, l, k}^{z(\downarrow)}=\bar{\rho}_{5 n, l, k}^{(\downarrow)}
\end{array}
$$

for any $\hat{a}_{n, l, k}^{(\uparrow, \downarrow) \dagger}|0\rangle$ and $\hat{b}_{n, l, k}^{(\uparrow, \downarrow) \dagger}|0\rangle$ states. We would emphasize that the above simple proportionality holds even beyond the LLLs.

The axial vector part is a little more complicated. After several line calculations, we arrive at the following expressions:

$$
\begin{aligned}
j_{5 n, l, k}^{z(\uparrow)}= & \frac{\left(\varepsilon_{n, l, k}^{(\uparrow)}+m\right)^{2}+k^{2}}{2 \varepsilon_{n, l, k}^{(\uparrow}\left(\varepsilon_{n, l, k}^{(\uparrow)}+m\right)}\left|\Phi_{n, l}\right|^{2} \\
& -\frac{|e| B(2 n+|l|+l+2)}{2 \varepsilon_{n, l, k}^{(\uparrow)}\left(\varepsilon_{n, l, k}^{(\uparrow)}+m\right)}\left|\Phi_{n, l+1}\right|^{2}
\end{aligned}
$$


for $\hat{a}_{n, l, k}^{(\uparrow) \dagger}|0\rangle$ states and

$$
\begin{aligned}
j_{5 n, l, k}^{z(\downarrow)}= & -\frac{\left(\varepsilon_{n, l, k}^{(\downarrow)}+m\right)^{2}+k^{2}}{2 \varepsilon_{n, l, k}^{(\downarrow)}\left(\varepsilon_{n, l, k}^{(\downarrow)}+m\right)}\left|\Phi_{n, l+1}\right|^{2} \\
& +\frac{|e| B(2 n+|l+1|+l+1)}{2 \varepsilon_{n, l, k}^{(\downarrow)}\left(\varepsilon_{n, l, k}^{(\downarrow)}+m\right)}\left|\Phi_{n, l}\right|^{2}
\end{aligned}
$$

for $\hat{a}_{n, l, k}^{(\downarrow \dagger}|0\rangle$ states. In the same way,

$$
\begin{aligned}
\bar{j}_{5 n, l, k}^{z(\uparrow)}= & \frac{\left(\bar{\varepsilon}_{n, l, k}^{(\uparrow)}+m\right)^{2}+k^{2}}{2 \bar{\varepsilon}_{n, l, k}^{(\uparrow)}\left(\bar{\varepsilon}_{n \uparrow l, k}^{(\uparrow)}+m\right)}\left|\Phi_{n,-l}\right|^{2} \\
& -\frac{|e| B(2 n+|l|-l)}{2 \bar{\varepsilon}_{n, l, k}^{(\uparrow)}\left(\bar{\varepsilon}_{n, l, k}^{(\uparrow)}+m\right)}\left|\Phi_{n,-l-1}\right|^{2}
\end{aligned}
$$

for $\hat{b}_{n, l, k}^{(\uparrow) \dagger}|0\rangle$ states and

$$
\begin{aligned}
\bar{j}_{5 n, l, k}^{z \downarrow)}= & -\frac{\left(\bar{\varepsilon}_{n, l, k}^{(\downarrow)}+m\right)^{2}+k^{2}}{2 \bar{\varepsilon}_{n, l, k}^{(\downarrow)}\left(\bar{\varepsilon}_{n, l, k}^{(\downarrow)}+m\right)}\left|\Phi_{n,-l-1}\right|^{2} \\
& +\frac{|e| B(2 n+|l+1|-l+1)}{2 \bar{\varepsilon}_{n, l, k}^{(\downarrow)}\left(\bar{\varepsilon}_{n, l, k}^{(\downarrow)}+m\right)}\left|\Phi_{n,-l}\right|^{2}
\end{aligned}
$$

for $\hat{b}_{n, l, k}^{(\downarrow) \dagger}|0\rangle$ states. One might think that the above expressions look similar to previous Eqs. (14) and (15), but the relative sign between two terms is different. Therefore, an elegant mode-by-mode relation like Eq. (25) cannot generally exist for the axial vector current.

Once we perform the spatial integration, we can sort out two terms into one, which yields

$$
\begin{aligned}
\mathfrak{j}_{5 n, l, k}^{z(\uparrow)} & =\int d x d y j_{5 n, l, k}^{z(\uparrow)} \\
& =\left[1-\frac{|e| B(2 n+|l|+l+2)}{\varepsilon_{n, l, k}^{(\uparrow)}\left(\varepsilon_{n, l, k}^{(\uparrow)}+m\right)}\right] \mathbf{r}_{n, l, k}^{(\uparrow)}
\end{aligned}
$$

and

$$
\begin{aligned}
\mathfrak{j}_{5 n, l, k}^{z(\downarrow)} & =\int d x d y j_{5 n, l, k}^{z(\downarrow)} \\
& =-\left[1-\frac{|e| B(2 n+|l+1|+l+1)}{\varepsilon_{n, l, k}^{(\downarrow)}\left(\varepsilon_{n, l, k}^{(\downarrow)}+m\right)}\right] \mathfrak{r}_{n, l, k}^{(\downarrow)} .
\end{aligned}
$$

Any further simplification is, however, impossible unless we limit ourselves to the LLLs. Indeed, for the LLLs only, a simple relation realizes as follows:

$$
j_{5 \mathrm{LLL}}^{z(\downarrow)}=-\rho_{\mathrm{LLL}}^{(\downarrow)}, \quad \bar{j}_{5 \mathrm{LLL}}^{z(\uparrow)}=-\bar{\rho}_{\mathrm{LLL}}^{(\uparrow)}
$$

for any $m$, for the second terms dropoff. We note that only the $(\downarrow)$ states have the LLLs for negatively charged particles and there is no counterpart relation for the ( $\uparrow)$ particle states. The same can be said about the antiparticle LLL states.

\section{APPLICATION TO THE CHIRAL MAGNETIC AND RELATED EFFECTS}

The CME is characterized by a finite vector current induced by a nonzero chirality under external magnetic fields. The most compact representation of the CME relies on the chiral chemical potential, $\mu_{5}$ and the formula is $\boldsymbol{j}=$ $\mu_{5} /\left(2 \pi^{2}\right) e \boldsymbol{B}$ (where our $\boldsymbol{j}$ is not an electric current but a vector current). Theoretically speaking, it would be desirable to define the CME without resorting to $\mu_{5}$ that is a troublesome object. A common alternative is a parity-odd background $\sim \boldsymbol{E} \cdot \boldsymbol{B}$ and, here, we are proposing a novel picture of the CME by means of the mode decomposition.

\section{A. Recovery of the chiral magnetic effect}

We first discuss how to derive the well-known formula of the ordinary CME with $\mu_{5}$ within the present framework. In the presence of $\mu_{5}$, the wave functions we presented before are no longer eigenstates and the eigenenergies should be reconsidered. Generally speaking, $\mu_{5}$ dependence is involved (but known; see Ref. [8]), and yet, the (,$\downarrow)$ parts give chirality contributions with equal weights due to degeneracy with incremented $n$ and conversion by $k \rightarrow-k$. Then, the current contributions cancel out due to an extra minus sign in Eq. (25), so that only the LLL contribution survives, as is the case in the standard CME computation. Now, we further simplify the calculation by taking the $m \rightarrow 0$ limit. We see that the LLL states have definite chirality for $m=0$ and we do not have to rediagonalize the Hamiltonian. Taking the spatial average, $\left(1 / S_{\perp}\right) \int d x d y$, we can then express the total current as

$$
\begin{aligned}
j^{z}= & j_{\mathrm{LLL}}^{z(\downarrow)}+\bar{j}_{\mathrm{LLL}}^{z(\uparrow)}=-\rho_{5, \mathrm{LLL}}^{(\downarrow)}-\bar{\rho}_{5, \mathrm{LLL}}^{(\uparrow)} \\
= & -\left(\frac{1}{S_{\perp}} \frac{2 \pi}{|e B|}\right) \frac{|e B|}{2 \pi}\left(\frac{S_{\perp}|e B|}{2 \pi}\right) \int \frac{d k}{2 \pi} \\
& \times\left[-\operatorname{sgn}(k) \theta\left(\mu_{5} \operatorname{sgn}(k)-|k|\right)\right. \\
& \left.+\operatorname{sgn}(k) \theta\left(-\mu_{5} \operatorname{sgn}(k)-|k|\right)\right] \\
= & \frac{|e B| \mu_{5}}{2 \pi^{2}}
\end{aligned}
$$

where the factor in the first parentheses comes from the spatial average on the wave functions, the second factor by $|e B| /(2 \pi)$ is from the phase space weight in Eq. (13), and the third factor is from the $J_{z}$ sum (degeneracy). We again note that we explicitly took the $m=0$ limit just for computational simplicity, but the CME itself is insensitive to $m$. 


\section{B. Mode decomposed chiral magnetic effect}

Next, we shall discuss the mode decomposed realization of the CME, which is one of the central results in this work. Before that, here, let us point out that Eq. (25) explains why we could find no simple relation between $j^{z}$ and $\rho_{5}$ until we make the helicity decomposition. That is, according to Eq. (25), $j_{n, l, k}^{z}=j_{n, l, k}^{z(\uparrow)}+j_{n, l, k}^{z(\downarrow)}=\rho_{5 n, l, k}^{(\uparrow)}-\rho_{5 n, l, k}^{(\downarrow)}$, while the chirality is $\rho_{5 n, l, k}=\rho_{5 n, l, k}^{(\uparrow)}+\rho_{5 n, l, k}^{(\downarrow)}$, and they cannot be equated. Thus, the $(\uparrow, \downarrow)$ projection is essential to establish such a simple relation.

Historically, the CME was first addressed in terms of $\rho_{5}$ in Ref. [6] (denoted differently in the original literature) in the context of the heavy-ion collisions, and then $\rho_{5}$ gave way to $\mu_{5}$ since Ref. [8], and now we are turning back to the original description with $\rho_{5}$. The point is that data from the heavy-ion collisions are convoluted with all time evolution and spatial fluctuations and the helicity decomposed measurement is unfeasible. With electron vortex beams, e.g., however, it may be possible to prepare wave functions with either $(\uparrow)$ or $(\downarrow)$ projected out. If we do so, we do no longer have to introduce $\mu_{5}$ but we can simply refer to Eq. (25) as the CME; more specifically to distinguish from the conventional CME, we may well call our Eq. (25) the mode decomposed chiral magnetic effect.

Let us reiterate the key expressions

$$
j_{n, l, k}^{z(\uparrow)}=\rho_{5 n, l, k}^{(\uparrow)}, \quad j_{n, l, k}^{z(\downarrow)}=-\rho_{5 n, l, k}^{(\downarrow)}
$$

for the particle states. It is worth emphasizing that this relation is not only for the LLL states but for any $(n, l, k)$ and any $m$. This feature makes a sharp contrast to the ordinary CME in which only the LLL contribution survives, as we already discussed. We would stress that this is a great advantage experimentally since Eq. (34) holds for arbitrary (even vanishing!) magnetic field and fermion mass. In other words, in the ordinary CME, the role of the magnetic field is to separate $(\uparrow, \downarrow)$ states. Therefore, the magnetic field would be anyway needed for such a purpose of the helicity decomposition in real setups.

One might think that Eq. (34) invokes an analogy to the $(1+1)$-dimensional system, i.e., the Dirac matrices algebraically satisfy

$$
\gamma^{\mu} \gamma_{5}=-\varepsilon^{\mu \nu} \gamma_{\nu}
$$

in $(1+1)$-dimensional theories, where our convention is $\varepsilon^{01}=-\varepsilon^{10}=1$. Then, the above identity is immediately translated into

$$
j_{\mathrm{LLL}}^{z}=-\rho_{5 \mathrm{LLL}}, \quad j_{5 \mathrm{LLL}}^{z}=-\rho_{\mathrm{LLL}}
$$

for the effectively $(1+1)$-dimensional LLLs in the case with negatively charged particles. These relations, however, make sense only when the magnetic field is strong enough to justify the LLL approximation. In this sense, Eq. (34) keeps more physical contents including higher LL correspondence.

\section{Contrast to the chiral separation effect}

Now, it would be instructive to consider the CSE here. Unlike the CME, the CSE is mass dependent, which was recognized since one of the earliest works [22]. In the CSE, an axial vector current arises in finite-density matter in response to an external magnetic field. The CSE formula takes a simple form only in the massless limit, and in fact, our Eqs. (30) and (31) can get simplified for the $m=0$ case only as

$$
\mathrm{i}_{5 n, l, k}^{z(\uparrow)}=\frac{k^{2}}{\varepsilon_{n, l, k}^{(\uparrow) 2}} \mathfrak{r}_{n, l, k}^{(\uparrow)}, \quad \dot{\mathrm{i}}_{5 n, l, k}^{z(\downarrow)}=-\frac{k^{2}}{\varepsilon_{n, l, k}^{(\downarrow) 2}} \mathfrak{r}_{n, l, k}^{(\downarrow)} .
$$

In the same way as the CME derivation, with incremented $n$, the energy dispersion relations for $(\uparrow, \downarrow)$ become degenerated at finite $\mu$, and only the current contributions from the LLLs survive. From almost the same procedure as Eq. (33), using Eq. (32), we can recover the CSE as follows:

$$
\begin{aligned}
j_{5}^{z} & =j_{5, \mathrm{LLL}}^{z(\downarrow)}+\bar{j}_{5, \mathrm{LLL}}^{z(\uparrow)}=-\rho_{\mathrm{LLL}}^{(\downarrow)}-\bar{\rho}_{\mathrm{LLL}}^{(\uparrow)} \\
& =-\frac{|e B|}{2 \pi} \int \frac{d k}{2 \pi} \theta(\mu-|k|)=-\frac{|e B| \mu}{2 \pi^{2}} .
\end{aligned}
$$

In this case, unlike Eq. (33), there is no contribution from the antiparticle at zero temperature. The overall minus sign is intuitively understandable; the axial vector current is identifiable with the spin, and the particle LLL states have the spin antiparallel to the magnetic field as sketched in Fig. 1.

\section{APPLICATION TO ROTATING FERMIONS}

The mode decomposed formulation turns out to be insightful for us to acquire intuitive understanding for rotational systems as well. We shall demonstrate concrete calculations and develop a perspective different from the ordinary method. To this end, first, we make a quick overview of the conventional field-theoretical treatment of rotating fermions. In the rotating frame, the Hamiltonian is modified into a cranking form, which is analogous to finitedensity effects as pointed out in Ref. [53]. Bearing this analogy in mind, we will introduce a working concept of grand canonical and canonical formulation of rotation.

\section{A. Rotating fermions}

In the rotating frame, we should incorporate a nontrivial metric as well as vierbeins for spinors. The covariant derivative in the Dirac equation then involves the spin connection. After some calculations, we can find that the free Dirac equation is given simply by 


$$
\left[\gamma^{0}\left(i D_{0}-\boldsymbol{\omega} \cdot \hat{\boldsymbol{J}}\right)-\boldsymbol{\gamma} \cdot i \boldsymbol{D}-m\right] \psi=0
$$

where the covariant derivative is $D_{\mu}=\partial_{\mu}+i e A_{\mu}$ with our convention $e<0$ (for electrons), and $\left(\gamma^{0}, \gamma\right)$ are defined in Dirac representation. Here, $\hat{\boldsymbol{J}}$ is the total angular momentum operator defined as $\hat{\boldsymbol{J}}=\hat{\boldsymbol{l}}+\hat{\boldsymbol{s}}=\boldsymbol{x} \times i \boldsymbol{\nabla}+\boldsymbol{\Sigma}$ using the spin matrix, $\boldsymbol{\Sigma}=\frac{i}{4} \boldsymbol{\gamma} \times \boldsymbol{\gamma}$. In the above, $\boldsymbol{\omega}$ denotes the angular velocity vector, which is taken along the $z$ direction, i.e., $\boldsymbol{\omega}=\omega \boldsymbol{e}_{z}$, without loss of generality.

\section{B. Grand canonical vs canonical formulations of rotation}

The modification in Eq. (39) clearly indicates that the one-particle energy, $\varepsilon_{n, l, k}^{(\uparrow, \downarrow)}$ is shifted by a rotation-induced "chemical potential" as

$$
\varepsilon_{n, l, k}^{(\uparrow, \downarrow)} \rightarrow \varepsilon_{n, l, k}^{(\uparrow, \downarrow)}-\mu_{\mathrm{eff} l}, \quad \mu_{\mathrm{eff} l}=\boldsymbol{\omega} \cdot \boldsymbol{J}=\omega\left(l+\frac{1}{2}\right) .
$$

It is quite natural that a finite $\omega>0$ energetically favors states with larger $J_{z}>0$. In many-body systems, the total energy $E_{\text {total }}$ would be thus shifted as

$$
E_{\text {total }} \rightarrow E_{\text {total }}-\omega J_{\text {total }},
$$

where $J_{\text {total }}$ represents the total angular momentum. Such an energy shift is reminiscent of the situation at finite chemical potential, i.e.,

$$
E_{\text {total }} \rightarrow E_{\text {total }}-\mu N_{\text {total }} .
$$

Here, $N_{\text {total }}$ is the total particle number. We see prominent similarity between Eqs. (41) and (42). This finite-density analogy of the rotational effect has been already pointed out in Ref. [53], which has inspired a phase diagram with the finite-density axis replaced with the angular velocity [54].

For finite-density systems, we know that $\mu>0$ in the grand canonical ensemble plays a role of pressure to induce a finite expectation value of density, i.e., $\left\langle N_{\text {total }}\right\rangle>0$. Alternatively, we can adopt the canonical ensemble picture and realize a finite-density system by forcing $N_{\text {total }}>0$ directly. In the thermodynamic limit (i.e., the limit of infinite volume and particle number), the grand canonical and the canonical descriptions should be completely equivalent.

For the rotating fermionic system, the above correspondence of $\mu \rightarrow \omega$ and $N_{\text {total }} \rightarrow J_{\text {total }}$ implies a canonical alternative with which $J_{\text {total }}$ is directly imposed instead of using $\omega \neq 0$. In fact, in finite size systems like nuclei treated in nuclear structure theory, it is a common and wellestablished method to deal with rotation of deformed nuclei by performing the angular momentum projection. Indeed, the angular momentum projection is required to restore rotational symmetry spontaneously broken by deformation.
This analogy to the canonical ensemble is, however, not perfect and there are several crucial differences. The most important difference comes from the fact that we cannot take the thermodynamic limit for rotating systems; otherwise, the causality bound would be violated. The next important difference is that the one particle energy shift in Eq. (40) is not a constant but changes with $J_{z}$, while the density shift is just a constant by $\varepsilon \rightarrow \varepsilon-\mu$. Since the fermion occupation should be one per phase space, fermions occupy low energy states up to $\varepsilon<\mu$. In contrast, in the rotational case, such occupied states with $\varepsilon_{n, l, k}^{(\uparrow, \downarrow)}<$ $\omega J_{z}$ are not necessarily limited to low energy states but high angular momentum states may be possibly accumulated near the boundary edges of the finite size system [44]. Therefore, unlike the finite-density case, the canonical approach to rotating fermionic systems may exhibit qualitative differences from what is expected from the conventional grand canonical description at $\omega \neq 0$. Still, a superposition of all canonical states with appropriate $(\omega$ dependent) weight factors would recover the conventional results obtained in the rotating frame at $\omega \neq 0$.

\section{Rotation-induced density}

As an example to show how to utilize our expressions for rotating fermions, let us consider the following problem. Without rotation and chemical potential, the particle and the antiparticle contributions cancel out in Eq. (12). The interesting question is what would happen for $\omega \neq 0$ and $e B \neq 0$ ? Then, for the particles and the antiparticles, an effective chemical potential is induced and the distribution functions should be $\theta\left(\omega J_{z}-\varepsilon_{n, l, k}^{(\uparrow, \downarrow)}\right)$ and $\theta\left(\omega J_{z}-\bar{\varepsilon}_{n, l, k}^{(\uparrow, \downarrow)}\right)$, respectively, at zero temperature.

For the calculation of the density $\rho$, as discussed in Ref. [45], only the LLL contribution remains finite up to the linear order in $\omega$. For the LLLs, then, $J_{z}<0$ for $\omega>0$ is chosen out for the negatively charged particles, and $J_{z}>0$ is for the antiparticles. It is clear from the above stepfunction distribution functions that only the antiparticle LLL states make nonzero contributions. Therefore, the density is attributed solely to the antiparticle LLL states with $\omega J_{z}>0$, and this is along the direction of the antiparticle LLL motion as illustrated in Fig. 1. To summarize, for $m=0$, we can express the spatially averaged density as follows:

$$
\begin{aligned}
\rho & =-\left(\frac{1}{S_{\perp}} \frac{2 \pi}{|e B|}\right) \frac{|e B|}{2 \pi} \sum_{J_{z}>0}^{S_{\perp}|e B| /(2 \pi)} \int \frac{d k}{2 \pi} \theta\left(\omega J_{z}-|k|\right) \\
& =-\frac{\omega}{\pi S_{\perp}} \sum_{J_{z}}^{S_{\perp}|e B| /(2 \pi)}\left(l+\frac{1}{2}\right) \\
& =-\frac{\omega|e B|}{4 \pi^{2}}+\text { (orbital part). }
\end{aligned}
$$


Therefore, the spin contribution to an induced density reads

$$
\rho_{\text {spin }}=-\frac{\omega|e B|}{4 \pi^{2}},
$$

which correctly coincides with Ref. [45]. The orbital part may look proportional to $S_{\perp}$ which diverges in the thermodynamic limit. This pathological behavior arises from the magnetic contribution to the angular momentum, and we need careful treatments of the canonical and the kinetic orbital angular momenta [81]. They are different by $|e B| r^{2} / 2$ [82] and this extra contribution (coming from the Poynting vector) cancels the orbital part. We will present more detailed and explicit discussions on this subtle but interesting point in another publication.

\section{Chiral vortical effect}

The CVE is characterized by an axial vector current induced in matter at $\omega \neq 0$. The current can exist at finite value of either temperature or density, and we will consider the finite-density situation only in this paper. Then, the CVE formula should be $\boldsymbol{j}_{5}=\mu^{2} /\left(2 \pi^{2}\right) \boldsymbol{\omega}$ in the case of $m=0$ and $|e B|=0$. The coexistence of finite magnetic field may change the formula, and it would be an intriguing question to generalize the CVE formula to the finite magnetic case. The extra magnetic contribution to the orbital angular momentum is, however, a subtle problem and we will discuss it in another publication.

In the massless limit, the axial vector current (after the spatial average) is given by

$$
\begin{aligned}
j_{5}^{z}= & \left(\frac{1}{S_{\perp}} \frac{2 \pi}{|e B|}\right) \frac{|e B|}{2 \pi} \sum_{n, l} \int \frac{d k}{2 \pi}\left[\frac{k^{2}}{\varepsilon_{n, l, k}^{(\uparrow) 2}} \theta\left(\omega J_{z}+\mu-\varepsilon_{n, l, k}^{(\uparrow)}\right)\right. \\
& -\frac{k^{2}}{\varepsilon_{n, l, k}^{(\downarrow) 2}} \theta\left(\omega J_{z}+\mu-\varepsilon_{n, l, k}^{(\downarrow)}\right)+\frac{k^{2}}{\bar{\varepsilon}_{n, l, k}^{(\uparrow) 2}} \theta\left(\omega J_{z}-\mu-\bar{\varepsilon}_{n, l, k}^{(\uparrow)}\right) \\
& \left.-\frac{k^{2}}{\bar{\varepsilon}_{n, l, k}^{(\downarrow) 2}} \theta\left(\omega J_{z}-\mu-\bar{\varepsilon}_{n, l, k}^{(\downarrow)}\right)\right] .
\end{aligned}
$$

One might think that only the LLLs remain with incremented $n$, but in this case $J_{z}$ enters the step functions and the cancellation is incomplete. Namely, the LLLs give

$$
\begin{aligned}
j_{5, \mathrm{LLL}}^{z}= & \frac{1}{S_{\perp}}\left[-\sum_{J_{z}<0}^{-S_{\perp}|e B| /(2 \pi)} \int \frac{d k}{2 \pi} \theta\left(\omega J_{z}+\mu-|k|\right)\right. \\
& \left.+\sum_{J_{z}>0}^{S_{\perp}|e B| /(2 \pi)} \int \frac{d k}{2 \pi} \theta\left(\omega J_{z}-\mu-|k|\right)\right] \\
= & (\omega-2 \mu) \frac{|e B|}{4 \pi^{2}}+\text { (orbital part) },
\end{aligned}
$$

and the first term is completely consistent with a combination of the LLL relation (36) and the induced density (44). The second term is nothing but the CSE formula (38).

For higher LL contributions, we note that $\varepsilon_{n, l, k}^{(\uparrow)}=$ $\varepsilon_{n+1, l-1, k}^{(\downarrow)}$ and $\bar{\varepsilon}_{n, l, k}^{(\downarrow)}=\bar{\varepsilon}_{n+1, l+1, k}^{(\uparrow)}$. By incrementing $n$ and shifting $l$ accordingly, we get

$$
\begin{aligned}
j_{5}^{z} & =\frac{1}{S_{\perp}} \sum_{n>0, l} \int \frac{d k}{2 \pi}\left\{\frac{k^{2}}{\varepsilon_{n, l, k}^{(\downarrow) 2}}\left[\theta\left(\omega J_{z}+\omega+\mu-\varepsilon_{n, l, k}^{(\downarrow)}\right)-\theta\left(\omega J_{z}+\mu-\varepsilon_{n, l, k}^{(\downarrow)}\right)\right]+\frac{k^{2}}{\bar{\varepsilon}_{n, l, k}^{(\uparrow) 2}}\left[\theta\left(\omega J_{z}-\mu-\bar{\varepsilon}_{n, l, k}^{(\uparrow)}\right)-\theta\left(\omega J_{z}-\omega-\mu-\bar{\varepsilon}_{n, l, k}^{(\uparrow)}\right)\right]\right\} \\
& =\frac{1}{S_{\perp}} \sum_{n>0, l} \int \frac{d k}{2 \pi} \frac{k^{2}}{\varepsilon_{n, l, k}^{(\downarrow) 2}}\left[\theta\left(\omega J_{z}+\omega+\mu-\varepsilon_{n, l, k}^{(\downarrow)}\right)-\theta\left(\omega J_{z}+\mu-\varepsilon_{n, l, k}^{(\downarrow)}\right)+\theta\left(-\omega J_{z}-\mu-\varepsilon_{n, l, k}^{(\downarrow)}\right)-\theta\left(-\omega J_{z}-\omega-\mu-\varepsilon_{n, l, k}^{(\downarrow)}\right)\right] \\
& \simeq \frac{1}{S_{\perp}} \sum_{n>0, l} \int \frac{d k}{2 \pi} \frac{k^{2} \omega}{\varepsilon_{n, l, k}^{(\downarrow) 2}}\left[\delta\left(\mu-\varepsilon_{n, l, k}^{(\downarrow)}\right)+\delta\left(-\mu-\varepsilon_{n, l, k}^{(\downarrow)}\right)\right] .
\end{aligned}
$$

Here, in the last step, we made expansion in terms of $\omega \ll 1$, which is adopted in the standard derivation of the CVE formula. For $\mu>0$, the second term from the antiparticle is vanishing. The term involving $J_{z}$ is of higher order in the $\omega$ expansion. Then, in the limit of $e B \rightarrow 0$, we can replace $\left(1 / S_{\perp}\right) \sum_{n, l} \int d k_{\perp}^{2} /(2 \pi)^{2}$ [as we noted below Eq. (13)]. The phase space integration leads to

$$
j_{5}^{z} \simeq \omega \int \frac{d^{3} k}{(2 \pi)^{3}} \frac{k_{z}^{2}}{\varepsilon_{k}^{2}} \delta\left(\mu-\varepsilon_{k}\right)=\frac{\mu^{2} \omega}{6 \pi^{2}} .
$$

One may think that this result has a wrong coefficient by $1 / 3$ as compared to the CVE formula, but the calculation is completely correct and this factor $1 / 3$ problem has been already resolved well.

The remaining $2 / 3$ contributions are accounted for by the magnetization current. The magnetization current is of great importance to maintain Lorentz symmetry [42,83-85], which is also associated with the side-jump effect [86-88]. For recent discussions on the side-jump effect within the framework of the chiral kinetic theory, see Refs. [89-92].

The most interesting observation in the calculation of Eq. (47) is that the energy derivative appears in the small $\omega$ limit from a shift in $l$ for opposite $(\uparrow, \downarrow)$. This derivative is already seen in the original field-theoretical computation [25], but our mode decomposed description gives a plain 
explanation of the microscopic origin of the $\omega$ derivative. Now, we could have reproduced the correct CVE formula, but this requires extra discussions on the magnetic contribution to the angular momentum. In fact, it would be an extremely interesting question how the CVE formula would be modified by nonzero $e \boldsymbol{B}$. One might think that Eq. (47) already gives the answer, but even for numerical analysis, more careful treatments (e.g., imposing a proper boundary condition at $r=R$ to discretize the transverse momenta; see Ref. [46]) are crucial. Since these vital improvements are technically involved, and since this is a tremendously important problem on its own, we will report all such details in another publication.

\section{SUMMARY}

In this work, we introduced the mode decomposed description of fermionic wave functions at finite magnetic field $\boldsymbol{B}$. In this framework of the mode decomposed description, fermion fields are presented with quantum numbers corresponding to the helicity and the orbital angular momentum $l$. Using these wave functions, we obtained explicit forms of the mode-by-mode contributions to the density, the chirality density, the vector current, and the axial vector current. We found a remarkable relation between the vector current mode $j_{n, l, k}^{z(\uparrow, \downarrow)}$ and the chirality density $\rho_{5 n, l, k}^{(\uparrow, \downarrow)}$. We would call this surprisingly simple relation, $j_{n, l, k}^{z(\uparrow, \downarrow)}= \pm \rho_{5 n, l, k}^{(\uparrow, \downarrow)}$, the mode decomposed CME. We would emphasize noteworthy advantages of the mode decomposed CME as follows: first of all, not the chiral chemical potential but the chirality density is directly associated with the vector current. In this way, the mode decomposed CME can evade the problematic chiral chemical potential which may cause controversial interpretations. We confirmed that an appropriate superposition of modes weighted with the chiral chemical potential can correctly reproduce the usual CME from the mode decomposed formula. Second of all, the mode decomposed CME does hold for all Landau levels and any mass. We note that a similar relation between the axial current and the density is derived only for the LLLs. Such an elegant relation reminds us that the CME is anomaly protected, i.e., the coefficient in front of the magnetic field is unaffected by any infrared scales. It would be an intriguing future problem to study how the mode decomposed CME may undergo a change by interaction effects. Probably only the LLL part is anomaly protected as the CME is, and yet, the mode decomposed CME makes sense as long as the quasiparticle picture is valid (e.g., in the mean-field approximation in which interaction effects are renormalized in a dressed mass).

Furthermore, we applied the mode decomposed formulation to rotating fermion systems. It is known that a finite rotation shifts the one-particle energy by a cranking term, $\boldsymbol{\omega} \cdot \boldsymbol{J}$, which has analogy with the $\mu N$ term in finite-density systems. In our mode decomposed formulation, a mode with a fixed $l$ is specifically considered, and our analysis, in this sense, is regarded as the canonical approach in which $N$ for finite density is directly fixed. The grand canonical approach fixes a finite $\mu$ for finite density and a finite $\omega$ for finite rotation. We note that the canonical approach to fix $l$ and the grand canonical approach to fix $\omega$ cannot be equivalent since the causality bound prevents us from taking the thermodynamic limit. One application of the mode decomposed description is the analysis on the density redistribution $\propto \boldsymbol{\omega} \cdot \boldsymbol{B}$ as found in Ref. [45]. The favored LLLs at finite $\boldsymbol{B}$ for particles and antiparticles are directed in opposite orientations, and a finite rotation induces imbalance between particles and antiparticles. We also checked that the CVE formula apart from the magnetization current results from the weighted sum of higher Landau levels. Noncentral heavy-ion collisions produce hot and dense matter with strong magnetic field and huge vorticity. Our formulation can potentially have a tight connection to heavy-ion collisions at finite impact parameter.

In closing, let us mention a prospective possibility of implementing electron vortex beams. The mode decomposed formulation as presented in this paper is to be translated into the theory of electron vortex beams for which wave functions with fixed $l$ are concerned. Phase vortices are receiving increased attention in particle collision physics [62]. Applying our formulation to this topic could be an intriguing possibility. Furthermore, we would say that the idea could be directly tested in an optical setup; a particularly interesting example is the mode decomposed CME manifested by the helicity projection as discussed in this work. If relativistic electron beams with a certain helicity selected out are prepared, a possible realization of the mode decomposed CME should be the generation of finite chirality density in response to the imposed helicity projected current through Eq. (34). In other words, one can handle the chirality density by manipulating the helicity projected current and vice versa. This situation of electron beams makes a sharp contrast to that in heavy-ion collision experiments. In the latter, a finite chirality density could be created only locally in each collision event, while only averaged quantities such as fluctuations are measurable.

We would emphasize an attractive point of electron vortex beams. Now, nonrelativistic beams produce vortex beams with $l$ separated, and relativistic beams are going to be available in the near future. The impact is immense; it is still quite difficult to control rotation in experiments. The heavy-ion collision can certainly generate relativistic swirl, but rotation itself is not really controllable. It is even challenging to control rotation in tabletop experiments. A large $l$ instead of a large $\omega$ could be an alternative method to investigate rotating fermions. For the purpose to establish more reliable predictions for possible experiments especially when a finite magnetic field coexists, it is indispensable to develop a theoretical framework in which 
electromagnetic angular momenta are properly taken into account (see, e.g., Ref. [93] for significance of electromagnetic contributions). All these issues including optical applications for chiral physics deserve further investigations.

\section{ACKNOWLEDGMENTS}

We thank Xu-Guang Huang and Kazuya Mameda for useful discussions. K. F. was supported by Japan Society for the Promotion of Science KAKENHI Grants No. 18H01211 and No. 19K21874.
[1] S. L. Adler, Phys. Rev. 177, 2426 (1969).

[2] J. S. Bell and R. Jackiw, Nuovo Cimento A 60, 47 (1969).

[3] E. Witten, Nucl. Phys. B156, 269 (1979).

[4] G. Veneziano, Nucl. Phys. B159, 213 (1979).

[5] D. Kharzeev, R. Pisarski, and M. H. Tytgat, Phys. Rev. Lett. 81, 512 (1998).

[6] D. E. Kharzeev, L. D. McLerran, and H. J. Warringa, Nucl. Phys. A803, 227 (2008).

[7] A. Vilenkin, Phys. Rev. D 22, 3080 (1980).

[8] K. Fukushima, D. E. Kharzeev, and H. J. Warringa, Phys. Rev. D 78, 074033 (2008).

[9] D. E. Kharzeev and H. J. Warringa, Phys. Rev. D 80, 034028 (2009).

[10] B. I. Abelev et al. (STAR Collaboration), Phys. Rev. Lett. 103, 251601 (2009).

[11] B. Abelev et al. (STAR Collaboration), Phys. Rev. C 81, 054908 (2010).

[12] D. E. Kharzeev and D. T. Son, Phys. Rev. Lett. 106, 062301 (2011).

[13] B. Abelev et al. (ALICE Collaboration), Phys. Rev. Lett. 110, 012301 (2013).

[14] X.-G. Huang, Rep. Prog. Phys. 79, 076302 (2016).

[15] D. E. Kharzeev, J. Liao, S. A. Voloshin, and G. Wang, Prog. Part. Nucl. Phys. 88, 1 (2016).

[16] D. Son and B. Spivak, Phys. Rev. B 88, 104412 (2013).

[17] Q. Li, D. E. Kharzeev, C. Zhang, Y. Huang, I. Pletikosic, A. V. Fedorov, R. D. Zhong, J. A. Schneeloch, G. D. Gu, and T. Valla, Nat. Phys. 12, 550 (2016).

[18] X. Huang et al., Phys. Rev. X 5, 031023 (2015).

[19] Q. Li and D. E. Kharzeev, Nucl. Phys. A956, 107 (2016).

[20] P. Copinger, K. Fukushima, and S. Pu, Phys. Rev. Lett. 121, 261602 (2018).

[21] D. T. Son and A. R. Zhitnitsky, Phys. Rev. D 70, 074018 (2004).

[22] M. A. Metlitski and A. R. Zhitnitsky, Phys. Rev. D 72, 045011 (2005).

[23] G. Newman and D. Son, Phys. Rev. D 73, 045006 (2006).

[24] A. Vilenkin, Phys. Rev. D 20, 1807 (1979).

[25] A. Vilenkin, Phys. Rev. D 21, 2260 (1980).

[26] J. Erdmenger, M. Haack, M. Kaminski, and A. Yarom, J. High Energy Phys. 01 (2009) 055.

[27] D. T. Son and P. Surowka, Phys. Rev. Lett. 103, 191601 (2009).

[28] K. Landsteiner, E. Megias, and F. Pena-Benitez, Phys. Rev. Lett. 107, 021601 (2011).

[29] A. Flachi and K. Fukushima, Phys. Rev. D 98, 096011 (2018).
[30] R. Abramchuk, Z. V. Khaidukov, and M. A. Zubkov, Phys. Rev. D 98, 076013 (2018).

[31] D. Kharzeev and A. Zhitnitsky, Nucl. Phys. A797, 67 (2007).

[32] M. Stephanov and Y. Yin, Phys. Rev. Lett. 109, 162001 (2012).

[33] B. McInnes, Nucl. Phys. B911, 173 (2016).

[34] E. V. Gorbar, V. A. Miransky, I. A. Shovkovy, and X. Wang, Phys. Rev. D 88, 025025 (2013).

[35] E.-d. Guo and S. Lin, J. High Energy Phys. 01 (2017) 111.

[36] S. Lin and L. Yang, Phys. Rev. D 98, 114022 (2018).

[37] Z. Wang, X. Guo, S. Shi, and P. Zhuang, Phys. Rev. D 100, 014015 (2019).

[38] K. Fukushima, Prog. Part. Nucl. Phys. 107, 167 (2019).

[39] K. Tuchin, Adv. High Energy Phys. 2013, 490495 (2013).

[40] W.-T. Deng and X.-G. Huang, Phys. Rev. C 93, 064907 (2016).

[41] L. Adamczyk et al. (STAR Collaboration), Nature (London) 548, 62 (2017).

[42] D. T. Son and N. Yamamoto, Phys. Rev. D 87, 085016 (2013).

[43] K. Hattori, Y. Hidaka, and D.-L. Yang, Phys. Rev. D 100, 096011 (2019).

[44] S. Ebihara, K. Fukushima, and K. Mameda, Phys. Lett. B 764, 94 (2017).

[45] K. Hattori and Y. Yin, Phys. Rev. Lett. 117, 152002 (2016).

[46] H.-L. Chen, K. Fukushima, X.-G. Huang, and K. Mameda, Phys. Rev. D 96, 054032 (2017).

[47] M. N. Chernodub and S. Gongyo, Phys. Rev. D 96, 096014 (2017).

[48] Y. Liu and I. Zahed, Phys. Rev. D 98, 014017 (2018).

[49] Y. Liu and I. Zahed, Phys. Rev. Lett. 120, 032001 (2018).

[50] L. Wang and G. Cao, Phys. Rev. D 97, 034014 (2018).

[51] G. Cao and L. He, Phys. Rev. D 100, 094015 (2019).

[52] H.-L. Chen, X.-G. Huang, and K. Mameda, arXiv: 1910.02700 .

[53] H.-L. Chen, K. Fukushima, X.-G. Huang, and K. Mameda, Phys. Rev. D 93, 104052 (2016).

[54] Y. Jiang and J. Liao, Phys. Rev. Lett. 117, 192302 (2016).

[55] K. Y. Bliokh, M. R. Dennis, and F. Nori, Phys. Rev. Lett. 107, 174802 (2011).

[56] A. G. Hayrapetyan, O. Matula, A. Aiello, A. Surzhykov, and S. Fritzsche, Phys. Rev. Lett. 112, 134801 (2014).

[57] I. Bialynicki-Birula and Z. Bialynicka-Birula, Phys. Rev. Lett. 118, 114801 (2017).

[58] K. van Kruining, A. G. Hayrapetyan, and J. B. Götte, Phys. Rev. Lett. 119, 030401 (2017).

[59] A. Rajabi and J. Berakdar, Phys. Rev. A 95, 063812 (2017). 
[60] A. J. Silenko, P. Zhang, and L. Zou, Phys. Rev. Lett. 121, 043202 (2018).

[61] A. J. Silenko, P. Zhang, and L. Zou, Phys. Rev. Lett. 122, 063201 (2019).

[62] I. P. Ivanov, N. Korchagin, A. Pimikov, and P. Zhang, Phys. Rev. Lett. 124, 192001 (2020).

[63] K. Yu. Bliokh, Y. P. Bliokh, S. Savel'ev, and F. Nori, Phys. Rev. Lett. 99, 190404 (2007).

[64] K. Y. Bliokh, M. R. Dennis, and F. Nori, Phys. Rev. A 96, 023622 (2017).

[65] R. J. Ducharme, I. G. da Paz, and A. G. Hayrapetyan, arXiv:1812.04957.

[66] L. Allen, M. W. Beijersbergen, R. J. C. Spreeuw, and J. P. Woerdman, Phys. Rev. A 45, 8185 (1992).

[67] K. Y. Bliokh, J. Dressel, and F. Nori, New J. Phys. 16, 093037 (2014).

[68] S. M. Barnett, Phys. Rev. Lett. 118, 114802 (2017).

[69] M. Uchida and A. Tonomura, Nature (London) 464, 737 (2010).

[70] J. Verbeeck, H. Tian, and P. Schattschneider, Nature (London) 467, 301 (2010).

[71] B. J. McMorran, A. Agrawal, I. M. Anderson, A. A. Herzing, H. J. Lezec, J. J. McClelland, and J. Unguris, Science 331, 192 (2011).

[72] C. W. Clark, R. Barankov, M. G. Huber, M. Arif, D. G. Cory, and D. A. Pushin, Nature (London) 525, 504 (2015).

[73] J. Harris, V. Grillo, E. Mafakheri, G. C. Gazzadi, S. Frabboni, R. W. Boyd, and E. Karimi, Nat. Phys. 11, 629 (2015).

[74] V. Grillo, G. C. Gazzadi, E. Mafakheri, S. Frabboni, E. Karimi, and R. W. Boyd, Phys. Rev. Lett. 114, 034801 (2015).

[75] K. Y. Bliokh et al., Phys. Rep. 690, 1 (2017).

[76] E. Mafakheri, A. H. Tavabi, P.-H. Lu, R. Balboni, F. Venturi, C. Menozzi, G. C. Gazzadi, S. Frabboni, A. Sit,
R. E. Dunin-Borkowski, E. Karimi, and V. Grillo, Appl. Phys. Lett. 110, 093113 (2017).

[77] L. Wang, Y. Jiang, L. He, and P. Zhuang, Phys. Rev. C 100, 034902 (2019).

[78] L. Wang, Y. Jiang, L. He, and P. Zhuang, Phys. Rev. D 100, 114009 (2019).

[79] X.-1. Sheng, D. H. Rischke, D. Vasak, and Q. Wang, Eur. Phys. J. A 54, 21 (2018).

[80] R.-D. Dong, R.-H. Fang, D.-F. Hou, and D. She, Chin. Phys. C 44, 074106 (2020).

[81] This important observation was pointed out to us by Kazuya Mameda.

[82] C. R. Greenshields, R. L. Stamps, S. Franke-Arnold, and S. M. Barnett, Phys. Rev. Lett. 113, 240404 (2014).

[83] C. Manuel and J. M. Torres-Rincon, Phys. Rev. D 89, 096002 (2014).

[84] J.-Y. Chen, D. T. Son, M. A. Stephanov, H.-U. Yee, and Y. Yin, Phys. Rev. Lett. 113, 182302 (2014).

[85] J.-Y. Chen, D. T. Son, and M. A. Stephanov, Phys. Rev. Lett. 115, 021601 (2015).

[86] L. Berger, Phys. Rev. B 2, 4559 (1970).

[87] N. Nagaosa, J. Sinova, S. Onoda, A. H. MacDonald, and N. P. Ong, Rev. Mod. Phys. 82, 1539 (2010).

[88] D. Xiao, M.-C. Chang, and Q. Niu, Rev. Mod. Phys. 82, 1959 (2010).

[89] Y. Hidaka, S. Pu, and D.-L. Yang, Phys. Rev. D 95, 091901 (2017).

[90] Y. Hidaka, S. Pu, and D.-L. Yang, Phys. Rev. D 97, 016004 (2018).

[91] Y. Hidaka and D.-L. Yang, Phys. Rev. D 98, 016012 (2018).

[92] X.-G. Huang and A. V. Sadofyev, J. High Energy Phys. 03 (2019) 084.

[93] K. Fukushima and S. Pu, arXiv:2001.00359. 